\title{
Salvesen's Method for Added Resistance Revisited
}

\section{Amini-Afshar, Mostafa}

Published in:

Journal of Offshore Mechanics and Arctic Engineering

Link to article, DOI:

$10.1115 / 1.4050213$

Publication date:

2021

Document Version

Peer reviewed version

Link back to DTU Orbit

Citation (APA):

Amini-Afshar, M. (2021). Salvesen's Method for Added Resistance Revisited. Journal of Offshore Mechanics and Arctic Engineering, 143(5), [051902]. https://doi.org/10.1115/1.4050213

\section{General rights}

Copyright and moral rights for the publications made accessible in the public portal are retained by the authors and/or other copyright owners and it is a condition of accessing publications that users recognise and abide by the legal requirements associated with these rights.

- Users may download and print one copy of any publication from the public portal for the purpose of private study or research.

- You may not further distribute the material or use it for any profit-making activity or commercial gain

- You may freely distribute the URL identifying the publication in the public portal

If you believe that this document breaches copyright please contact us providing details, and we will remove access to the work immediately and investigate your claim 


\title{
Salvesen's Method for Added Resistance Revisited
}

\author{
Mostafa Amini-Afshar \\ Technical University of Denmark \\ Department of Mechanical Engineering \\ Section for Fluid Mechanics, Coastal \& Maritime Engineering \\ maaf@mek.dtu.dk
}

\begin{abstract}
Almost four years after the appearance of Salvesen-Tuck-Faltinsen (STF) strip theory [1], Salvesen in 1974 published his popular method for calculation of added resistance [2], [3]. His method is based on an exact near-field formulation, however he applied the long-wave and the weak-scatterer assumptions to present his approximate method using the integrated quantities (hydrodynamic and geometrical coefficients). Considering the available computational powers in the 1970s, both of these assumptions were absolutely justifiable. The intention of this paper is to disseminate the results of a recent study at the Technical University of Denmark, whereby the Salvesen's formulation has been revisited and the added resistance is computed from the original exact equation without invoking the weak-scatterer or the long-wave assumptions. This is performed using the solutions of the radiation and the scattering problems, obtained by a low-order Boundary Element Method and the two-dimensional free-surface Green function inside our in-house $S T F$ theory implementation [4]. The weak-scatterer assumption is then removed through a direct calculation of the $x$-derivatives of the velocity potentials and the normal vectors along the body. Knowing the velocity potentials over each panel, the long-wave assumption is also avoided by a piece-wise analytical integration of sectional Kochin function [5]. The presented results for 5 ship geometries testify that the correct treatment of the original equation is achieved only after both of the above-mentioned assumptions are removed. Implemented in this manner, Salvesen's method proves to be relatively more accurate and robust than has been generally perceived during all these years.
\end{abstract}

\section{Keywords}

Wave Drift Force, Added Resistance, Strip Theory, Salvesen's Method

\section{Introduction}

Alongside Computational Fluid Dynamics (CFD) [6, 7] and three-dimensional (3D) weakly or fully nonlinear potential flow models $[8,9,10,11]$, state-of-the-art methods for calculation of second-order wave drift force and added resistance also include Enhanced Unified Theory, [12, 13, 14, 15]. Considering the computational effort and accuracy of the results, this method which is based on Unified Slender-Body Theory [16, 17, 18, 19, 20, 21, 22, 23], the Kochin function and Maruo's method [24], [25], is extremely efficient and mature. The Unified Slender-Body theory considers a pure two-dimensional (2D) strip-theory solution, together with a solution which takes into account the interaction of the adjacent two-dimensional sections in the vicinity of the body (so-called the inner field). This interaction solution is calculated by a matching process between the solution in the inner-field and a three-dimensional solution in the outer-field, where the Laplace equation is solved subject to the free-surface and the radiation boundary conditions all in their 3D form. In fact this theory unifies "ordinary" slender body theory at low limit of the encounter frequency where the Froude-Krylov exciting forces are dominant, with strip theory which is applicable at high values of the encounter frequency.

Two popular and classic examples of the methods for calculation of wave drift force and added resistance using strip theory are the method of Gerritsma-Beukelman [26], and the method of Salvesen 
[2], [3]. The method by Gerritsma-Beukelman [26] is based on the far-field approach where the drift force is made proportional to the amplitude of the radiated waves (due to both body motion and scattering) in the far field. According to this method, and based on their own strip theory presented in [27], the far-field radiated energy can be calculated by a line integral over the length of the vessel. As mentioned in the Abstract, the Salvesen's method is formulated using the STF strip theory. He derives the original 3D formulation for the hydrodynamic force starting from the near-field pressure integration over the body surface. After some manipulation, the equation for the mean second order force is expressed with respect to 2 terms. One is the Kochin Function and incorporates the interaction of the incident wave with the disturbance waves, and the other is due to the interaction of the disturbance waves with each other. He adopts the weak-scatterer assumption and neglects the second part, but mentions that this assumption might lead to inaccurate results in zero-speed or beam- and near-beam cases. The Kochin Function part of the equation is computed by the long-wave approximations, and the formulation is derived in terms of the integrated quantities like added mass, damping and some geometrical coefficients. In the late 1970's there was also some research conducted to improve the methodologies for force calculation by strip theories. For example the work presented in [28] is an extension of the Gerritsma-Beukelman method to oblique seas. In other research, in 1976 Lin and Read in [29] aimed to complement Salvesen's method by incorporating the body-generated waves (disturbance waves). They derived another set of exact equation for calculation of mean secondorder forces, and presented the final formulations based on the Kochin Function inside of strip theory. Unfortunately no calculations were presented in that paper. Lin and Read in [29] mentioned that the results would be published in a follow-up paper, but to the knowledge of the author this has not taken place. This fact has been noted also by Salvesen [3] and Loukakis [28].

Similar to the Enhanced Unified Theory, the Kochin Function and Maruo's methodology can be applied inside the $S T F$ strip theory to obtain the added resistance without the above-mentioned assumptions. Clearly the results will not be as accurate as those from the Enhanced Unified Theory. However it can be implemented with relative ease since for the strip theory no matching is required between the inner-solutions and the outer-solution, and the added resistance can be expressed with respect to the 2D sectional Kochin Function. The details of this implementation are presented in a recent paper [30], where it is also shown how this methodology predicts the added resistance by $S T F$ strip theory more accurately than the approximate Salvesen's method in [2], [3].

In the present paper, and as an another step towards utilizing all capacities of strip theory for calculation of wave drift force and added resistance, it is demonstrated that in fact Salvesen's method performs significantly better when evaluated based on its original exact formulation compared to the widely used approximate form. An implementation of the STF theory is used, where both the radiation and the scattering problems are solved based on a low-order Boundary Element Method and the two-dimensional free-surface Green function [4]. The knowledge of the velocity potentials over the sections allows us to avoid the weak-scatterer and the long-wave assumptions. The former by a direct calculation of $x$-derivatives of the potentials and the normal vectors along the ship length, and the latter through a piece-wise exact integration over the two-dimensional sections. Calculations are then conducted for five ship geometries, and the results are compared with experiments and other reference solutions. The results confirm that the weak-scatterer part of the equation should not be neglected, even for a slender geometry like the classic Wigley hull. Moreover it is demonstrated that the Kochin Function, which has been computed by Salvesen using the long-wave approximations, should be calculated instead based on the direct integration of the velocity potentials over the 2D sections. This is necessary as the long-wave approximations are not applicable in a majority of cases. By adopting both of these strategies, it is shown that the original equation in its exact form is relatively powerful in predicting the added resistance and wave drift forces accurately. It is important here to mention two major contributions where Salvesen's method has been considered without the long-wave assumptions. Both of these works are due to Ming-Chung Fang in [31] and [32]. The first work is based on strip theory, and the second is based on the 3D Boundary Element Method. In both cases the disturbance velocity potentials are ignored, which means that he has also invoked the weak-scatterer assumption. No attempt has been made in these studies to calculate the body potential terms, and it is argued that they have no significant contribution to the final results. So the main novelty of the present paper is the clear demonstration of the influence of the disturbance potential terms on the 
wave drift force and the added resistance.

In the coming sections, first the theory and the relevant formulations are introduced then the numerical methods are described in some detail and finally the results, discussions and conclusions are presented.

\section{Theory}

Adopting potential-flow theory, the total velocity potential describing the flow field is expressed as

$$
\Phi(x, y, z, t)=-U x+\Re\left\{\left(\phi_{0}+\phi_{s}+\sum_{k=2}^{6} \xi_{k} \phi_{k}\right) e^{\mathrm{i} \omega t}\right\} .
$$

Here $U$ is the forward speed, $\omega$ is the encounter frequency, $\xi_{k}$ is the complex amplitude of the body motion in the $k$ th mode, and $\phi_{k}$ is the corresponding radiation velocity potential due to unit-amplitude motion. Note that in STF theory, the surge mode is neglected. The scattering potential is denoted by $\phi_{s}$, and the velocity potential of the incident wave $\phi_{0}$ with amplitude $A$, heading angle $\beta$, wave number in deep water $K=\omega_{0}^{2} / g=2 \pi / \lambda$, wave frequency $\omega_{0}$ and wave length $\lambda$ is defined by

$$
\phi_{0}=\frac{\mathrm{i} g A}{\omega_{0}} e^{K z} e^{-\mathrm{i} K(x \cos \beta+y \sin \beta)} .
$$

The acceleration due to gravity is shown as $g$. The relation between the wave frequency and the encounter frequency $\omega$ is defined by $\omega_{0}=\omega+K U \cos \beta$. See also Figure 1 for further notations. Inside the $S T F$ theory the 3D velocity potentials for the radiation modes are decomposed into speed-dependent and speed-independent parts as $\phi_{k}=\phi_{k}^{0}+U /(\mathrm{i} \omega) \phi_{k}^{U}$, with $\phi_{k}^{U}=0$ for $k=2,3,4, \phi_{5}^{U}=\phi_{3}^{0}$ and $\phi_{6}^{U}=-\phi_{2}^{0}$. The corresponding 2D sectional potentials are defined by $\psi_{2}, \psi_{3}, \psi_{4}, \psi_{5}=\psi_{3}(-x+U /(\mathrm{i} \omega))$ and $\psi_{6}=\psi_{2}(x-U /(\mathrm{i} \omega))$. Therefore only 3 velocity potentials are unknown. All these velocity potentials satisfy the Laplace equation $\nabla^{2} \psi_{k}=0$ and the zero-speed free-surface condition

$$
\omega^{2} \psi_{k}-g \frac{\partial \psi_{k}}{\partial z}=0
$$

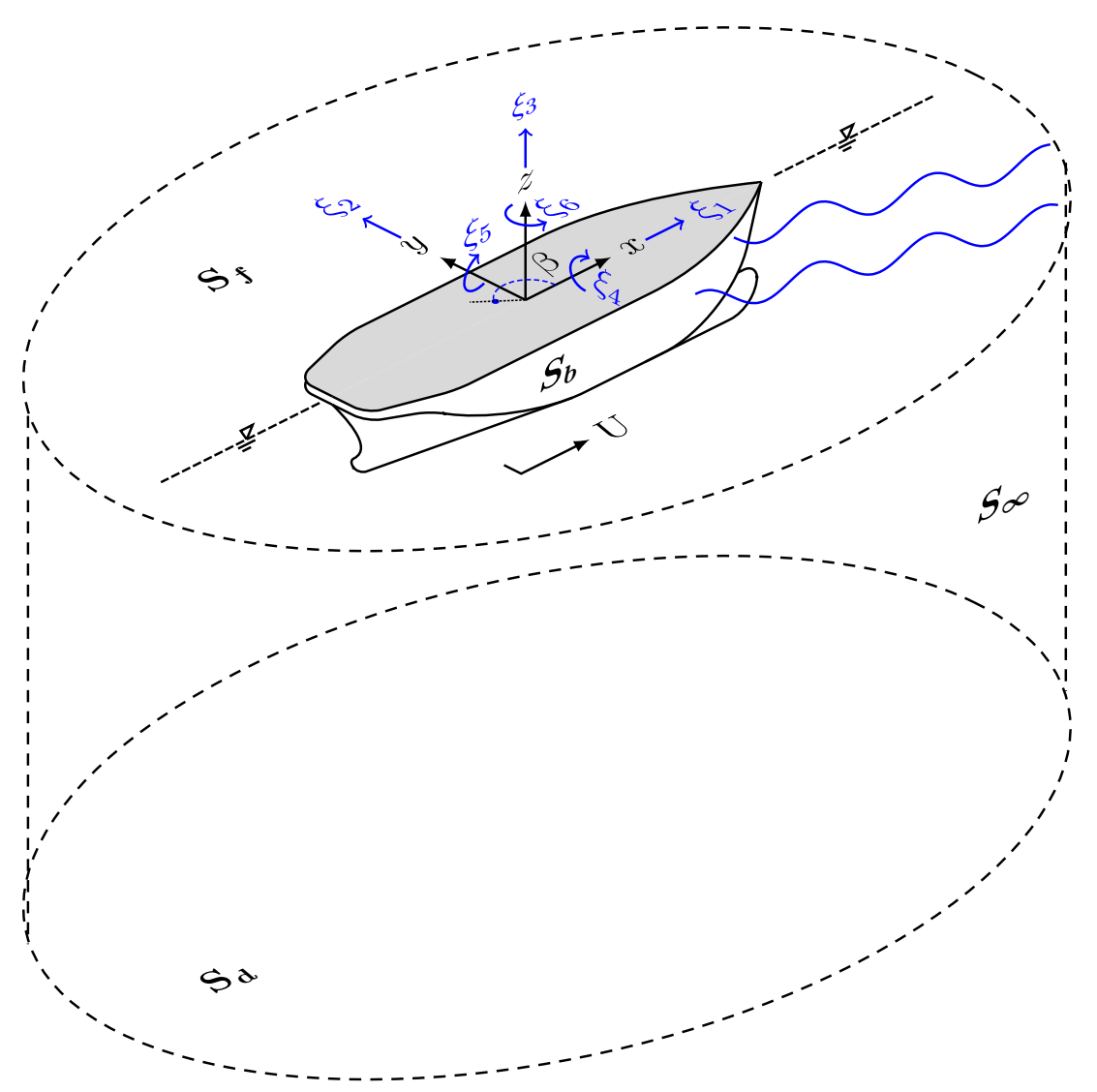

Figure 1: The sketch of the moving body and the flow domain. 
The body boundary condition for the radiation potentials are

$$
\frac{\partial \psi_{k}}{\partial N}=\mathrm{i} \omega N_{k}+U m_{k}
$$

where

$$
\begin{aligned}
\left(N_{1}, N_{2}, N_{3}\right) & =\boldsymbol{N}, \\
\left(N_{4}, N_{5}, N_{6}\right) & =\boldsymbol{r} \times \boldsymbol{N}, \\
\left(m_{1}, m_{2}, m_{3}\right) & =(0,0,0), \\
\left(m_{4}, m_{5}, m_{6}\right) & =\left(0, N_{3},-N_{2}\right) .
\end{aligned}
$$

Here $\boldsymbol{r}$ is the position vector and $\boldsymbol{N}$ is the 2D normal vector of points at the surface of the body $\boldsymbol{S}_{b}$, defined by

$$
N_{k}=\left(0, N_{2}, N_{3},\left(y N_{3}-z N_{2}\right),-x N_{3}, x N_{2}\right) .
$$

In addition, the sectional radiation velocity potentials should satisfy the radiation condition at the far field in the $y-z$ plane. In addition to the free-surface and the radiation conditions, the scattering velocity potential satisfies the body boundary condition

$$
\frac{\partial \psi_{s}}{\partial N}=-\frac{\partial \phi_{0}}{\partial N}
$$

For further details on the STF theory refer to [1].

In Appendix 1 of [2], Salvesen derives the expression for the hydrodynamic force, which is initiated by the near-field pressure integration over the body surface $\boldsymbol{S}_{b}$. Invoking Gauss's theorem for a volume enclosed by $\boldsymbol{S}_{b}, \boldsymbol{S}_{f}, \boldsymbol{S}_{\infty}$, Salvesen presents the following equation as the basis for calculation of the mean second-order wave forces

$$
\boldsymbol{F}(x, y, z, t)=\rho \iint_{\boldsymbol{S}_{\infty}}\left(\Phi_{B} \frac{\partial}{\partial n}-\frac{\partial \Phi_{B}}{\partial n}\right)\left[\nabla \Phi_{0}+\frac{1}{2} \nabla \Phi_{B}\right] d S .
$$

Note that $n$ denotes the $3 \mathrm{D}$ normal vector given by $\left(n_{1}, n_{2}, n_{3}\right)$. Here $\Phi_{B}=\Re\left\{\phi_{B} e^{\mathrm{i} \omega t}\right\}$ is the sum of the unsteady potentials due to all disturbance waves (radiation and scattering), and $\Phi_{0}=\Re\left\{\phi_{0} e^{\mathrm{i} \omega t}\right\}$ is the unsteady potential due to the incident wave. $\rho$ is the density of the fluid. This equation is comprised of the second-order pressure terms due to the product of the first-order velocity potentials. Typically each first-order quantity with harmonic time variation can be represented as $A_{j} \cos \left(\omega t+\theta_{j}\right)$, where the amplitude and the phase are denoted by $A_{j}$ and $\theta_{j}$ respectively. In the frequency domain, this quantity can be expressed by $\Re\left\{X_{j} e^{\mathrm{i} \omega t}\right\}$ in which $X_{j}=A_{j} e^{\mathrm{i} \theta_{j}}$ is the complex phasor. Therefore the second-order term $p^{(2)}$ arising from the product of two first-order quantities can be written as

$$
\begin{aligned}
p^{(2)} & =\left[A_{0} \cos \left(\omega t+\theta_{0}\right)\right]\left[A_{1} \cos \left(\omega t+\theta_{1}\right)\right]=\Re\left\{X_{0} e^{\mathrm{i} \omega t}\right\} \Re\left\{X_{1} e^{\mathrm{i} \omega t}\right\} \\
& =\left[\frac{X_{0}}{2} e^{\mathrm{i} \omega t}+\frac{X_{0}^{*}}{2} e^{-\mathrm{i} \omega t}\right]\left[\frac{X_{1}}{2} e^{\mathrm{i} \omega t}+\frac{X_{1}^{*}}{2} e^{-\mathrm{i} \omega t}\right] \\
& =\frac{1}{4}\left[X_{0} X_{1}^{*}+X_{1} X_{0}^{*}\right]+\frac{1}{4}\left(X_{0} X_{1} e^{2 \mathrm{i} \omega t}+X_{0}^{*} X_{1}^{*} e^{-2 \mathrm{i} \omega t}\right) \\
& =\frac{1}{4}\left[X_{0} X_{1}^{*}+X_{1} X_{0}^{*}\right]+\frac{1}{2} \Re\left\{X_{0} X_{1} e^{2 \mathrm{i} \omega t}\right\} .
\end{aligned}
$$

Here the asterisks * denote the complex conjugate. The time average of this second-order quantity over one wave period is then equal to

$$
\bar{p}^{(2)}=\frac{1}{4}\left(X_{0} X_{1}^{*}+X_{1} X_{0}^{*}\right) .
$$

This conclusion can be invoked, together with the complex phasors for the potentials of the disturbance and the incident wave $\left(\phi_{B}\right.$ and $\left.\phi_{0}\right)$, to calculate the added resistance as follows. First consider the interaction between the incident and the disturbance waves, i.e. only the part containing $\nabla \Phi_{0}$ on the 
right hand side of equation (2.6). Inserting the complex phasors of the velocity potentials, leads to the mean second-order force as

$$
\begin{aligned}
\overline{\boldsymbol{F}}_{\phi_{0}} & =\frac{1}{4} \rho \iint_{\boldsymbol{S}_{\infty}}\left\{\left(\phi_{B} \frac{\partial}{\partial n} \nabla \phi_{0}^{*}+\phi_{B}^{*} \frac{\partial}{\partial n} \nabla \phi_{0}\right)-\left(\nabla \phi_{0} \frac{\partial \phi_{B}^{*}}{\partial n}+\nabla \phi_{0}^{*} \frac{\partial \phi_{B}}{\partial n}\right)\right\} d S \\
& =\frac{1}{4} \rho \iint_{\boldsymbol{S}_{\infty}}\left\{\left(\phi_{B} \frac{\partial}{\partial n}-\frac{\partial \phi_{B}}{\partial n}\right) \nabla \phi_{0}^{*}+\left(\phi_{B}^{*} \frac{\partial}{\partial n}-\frac{\partial \phi_{B}^{*}}{\partial n}\right) \nabla \phi_{0}\right\} d S \\
& =\frac{1}{2} \rho \Re\left\{\iint_{\boldsymbol{S}_{\infty}}\left(\phi_{B} \frac{\partial}{\partial n}-\frac{\partial \phi_{B}}{\partial n}\right) \nabla \phi_{0}^{*} d S\right\} .
\end{aligned}
$$

In the same manner for $\nabla \Phi_{B}$ on the right hand side of Equation (2.6), which in fact represents the interaction between the disturbance waves, the mean second-order force can be expressed by

$$
\overline{\boldsymbol{F}}_{\phi_{B}}=\frac{1}{4} \rho \Re\left\{\iint_{\boldsymbol{S}_{\infty}}\left(\phi_{B} \frac{\partial}{\partial n}-\frac{\partial \phi_{B}}{\partial n}\right) \nabla \phi_{B}^{*} d S\right\} .
$$

For any pair of harmonic potentials $\phi_{i}, \phi_{j}$ (meaning $\nabla^{2} \phi_{i, j}=0$ ), which satisfy the free-surface condition, applying Green's theorem leads to

$$
I\left(\phi_{i}, \phi_{j}\right) \equiv \iint_{\boldsymbol{S}_{b}}\left(\phi_{i} \frac{\partial \phi_{j}}{\partial n}-\phi_{j} \frac{\partial \phi_{i}}{\partial n}\right) d S=-\iint_{\boldsymbol{S}_{\infty}}\left(\phi_{i} \frac{\partial \phi_{j}}{\partial n}-\phi_{j} \frac{\partial \phi_{i}}{\partial n}\right) d S .
$$

Note also that if both potentials satisfy the radiation condition, then the integral over $\boldsymbol{S}_{\infty}$ will vanish [33]. In equation (2.8) or (2.9), the disturbance potentials $\Phi_{B}$ satisfy all of the above-mentioned conditions. Except for the radiation condition, the same statement is also true in the case of $\nabla \Phi_{0}$ and $\nabla \phi_{B}^{*}$. Therefore the mean force can be expressed instead as an integral over the body surface

$$
\begin{aligned}
\overline{\boldsymbol{F}}_{\phi_{0}} & =-\frac{1}{2} \rho \Re\left\{\iint_{\boldsymbol{S}_{b}}\left(\phi_{B} \frac{\partial}{\partial n}-\frac{\partial \phi_{B}}{\partial n}\right) \nabla \phi_{0}^{*} d S\right\}, \\
\overline{\boldsymbol{F}}_{\phi_{B}} & =-\frac{1}{4} \rho \Re\left\{\iint_{\boldsymbol{S}_{b}}\left(\phi_{B} \frac{\partial}{\partial n}-\frac{\partial \phi_{B}}{\partial n}\right) \nabla \phi_{B}^{*} d S\right\} .
\end{aligned}
$$

It is important to mention that no strip theory assumptions are adopted in deriving the above equations, and they are expressed in their 3D form. Salvesen assumes that $\Phi_{B} \ll \Phi_{0}$, and accordingly neglects the second contribution $\overline{\boldsymbol{F}}_{\phi_{B}}$, which is a weak-scatterer assumption. Considering only (2.11) and inserting the incident wave potential he obtains

$$
\begin{aligned}
& \bar{F}_{x}^{\phi_{0}}=-\frac{\rho A g k \cos \beta}{2 \omega_{0}} \Re\left\{\int_{L} e^{\mathrm{i} K x \cos \beta}\left[\int_{C_{x}}\left(\psi_{B} \frac{\partial}{\partial N}-\frac{\partial \psi_{B}}{\partial N}\right) e^{K z} e^{\mathrm{i} K y \sin \beta} d l\right] d x\right\}, \\
& \bar{F}_{y}^{\phi_{0}}=-\frac{\rho A g k \sin \beta}{2 \omega_{0}} \Re\left\{\int_{L} e^{\mathrm{i} K x \cos \beta}\left[\int_{C_{x}}\left(\psi_{B} \frac{\partial}{\partial N}-\frac{\partial \psi_{B}}{\partial N}\right) e^{K z} e^{\mathrm{i} K y \sin \beta} d l\right] d x\right\},
\end{aligned}
$$

for the added resistance and the horizontal drift force respectively, within the STF strip theory. As mentioned before these equations are in fact the Kochin Function [5]. Here $\psi_{B}$ represents the sectional velocity potentials of the disturbance waves, and the integration over a $2 \mathrm{D}$ section is denoted by $C_{x}$. The body length is shown by $L$. In addition Salvesen adopts the following long-wave assumptions for computation of the sectional integrals of the Kochin Functions in (2.13) and (2.14)

$$
\begin{aligned}
e^{K z} & \approx e^{-K s d}, \\
e^{\mathrm{i} K y \sin \beta} & \approx e^{\mathrm{i} K\left( \pm \frac{1}{2} b\right) s \sin \beta},
\end{aligned}
$$

in which $b$ is the sectional beam, $d$ is the sectional draft, and $s$ is the sectional area coefficient calculated by normalizing the sectional area with $b d$. Adopting the Haskind relation, the added resistance and the mean drift force then is expressed with respect to line integrals along the body comprising only of the integrated quantities like sectional added mass, damping and the above-mentioned geometrical coefficients. See the details of this derivation and the final approximate formulations in Section (3.1) 
of his paper [2]. In a quite recent paper by the author [30], using the long-wave assumptions (2.15) and (2.16) the approximate relation for the Kochin Function are presented for calculation of the added resistance and drift force inside STF theory using Maruo's methodology [24], [25]. In that paper it is shown that these long-wave assumptions are only valid for zero- and very low speeds, and therefore for accurate results the Kochin Function should be computed directly based on the knowledge of the sectional velocity potentials $\psi_{B}$. Therefore it sounds logical to investigate the impact of these long-wave assumptions also on the approximate equations presented by Salvesen for calculation of (2.13) or (2.14). As mentioned in the introduction section, an implementation of the STF strip theory is performed where both the radiation and the scattering potentials are computed based on the Boundary Element Method and the 2D free-surface Green Function. See [4] and [30] for more details. This allows the Kochin Function in (2.13) or (2.14) to be computed directly, using a piecewise analytical integration over the two-dimensional section. As is shown in the results section, these long-wave assumptions break down in forward-speed cases also here for the calculation of the added resistance based on Salvesen's approximate formulations for (2.13) or (2.14).

Next the disturbance potential part $\overline{\boldsymbol{F}}_{\phi_{B}}$ in $(2.12)$ is treated. For the added resistance, the $x$ component of the force is

$$
\bar{F}_{x}^{\phi_{B}}=-\frac{1}{4} \rho \Re\left\{\iint_{S_{b}}\left(\phi_{B} \frac{\partial}{\partial n}-\frac{\partial \phi_{B}}{\partial n}\right) \frac{\partial \phi_{B}^{*}}{\partial x} d S\right\} .
$$

Since the sectional velocity potentials are known, this part is computed using a direct calculation of the $x$-derivatives of the velocity potentials and the normal vectors along the length of the body. Inserting the potentials and the body boundary conditions from their $2 \mathrm{D}$ definitions in STF theory, equation (2.17) in its expanded form is

$$
\bar{F}_{x}^{\phi_{B}}=-\frac{1}{4} \rho \Re\left\{\int_{L}\left(\int_{C_{x}}\left(W M^{*}-N Q^{*}\right) d l\right) d x\right\},
$$

in which

$$
\begin{aligned}
W & =\psi_{3} \xi_{3}+\psi_{3}(U / \mathrm{i} \omega-x) \xi_{5}+\psi_{s}, \\
Q & =\xi_{3} \psi_{3}^{x}+\xi_{5} \psi_{3}^{x}(U / \mathrm{i} \omega-x)-\xi_{5} \psi_{3}+\psi_{s}^{x}, \\
N & =\mathrm{i} \omega \xi_{3} N_{3}+\xi_{5}\left(U N_{3}-\mathrm{i} \omega x N_{3}\right)-K \phi_{0}\left(N_{3}-\mathrm{i} N_{2} \sin \beta\right), \\
M & =\mathrm{i} \omega \xi_{3} N_{3}^{x}+U \xi_{5} N_{3}^{x}-\mathrm{i} \omega x \xi_{5} N_{3}^{x}-\mathrm{i} \omega \xi_{5} N_{3}-K \phi_{0}^{x}\left(N_{3}-\mathrm{i} N_{2} \sin \beta\right)-K \phi_{0}\left(N_{3}^{x}-\mathrm{i} N_{2}^{x} \sin \beta\right) .
\end{aligned}
$$

Here the superscript ${ }^{x}$ denotes the $x$-derivative. The results shown in the next section confirm that in fact the body disturbance term is an important part of Equation (2.6), and has a profound effect on the added resistance. In the results section, it is demonstrated that a direct computation of the Kochin Function in (2.13) is not enough to get accurate solutions. It is only after incorporating Equation (2.18) and treating (2.6) as a whole that accurate results are achieved. The reason that Salvesen's approximate method provides reasonable results is that the errors introduced by the longwave assumption largely cancel the neglect of the disturbance potential terms. This behavior is also shown in the result section.

\section{Numerical Methods}

In this section some details of the numerical methods are described. An in-house implementation of the $S T F$ theory is used [4], whereby the solutions for the radiation and the scattering potentials are provided for each two-dimensional section along the ship length. See [30] for further details.

\subsection{Evaluation of the Kochin Function Term}

According to the Boundary Element Method implemented for the $S T F$ strip theory in [4], $\psi_{B}$ is calculated and known. Having the geometry of each panel including $N_{2}, N_{3}$, and replacing the normal derivative of $\psi_{B}$ with the body boundary condition from (2.4) or (2.5), the line integrals over $C_{x}$ in 
(2.13) or (2.14) can be computed. As the velocity potential and the normal vector are assumed to be constant over each panel, these integrals can be evaluated easily by a series of piece-wise exact integrations along all panels. So the part of the sectional integral related to the gradient of the potentials can be calculated as

$$
I_{1}=\sum_{m=1}^{N_{p}-1} \frac{\mathrm{i}}{K(\sin \delta+\mathrm{i} \sin \beta \cos \delta)}\left\{e^{K z+\mathrm{i} K y \sin \beta}\right\}_{m}^{m+1},
$$

and the part related to the velocity potential can be calculated as

$$
I_{2}=\sum_{m=1}^{N_{p}-1} \frac{-\cos \delta+\mathrm{i} \sin \beta \sin \delta}{\sin \delta+\mathrm{i} \sin \beta \cos \delta}\left\{e^{K z+\mathrm{i} K y \sin \beta}\right\}_{m}^{m+1},
$$

in which $\delta$ is the angle of the normal vector to each panel $\left(N_{2}=\sin \delta, N_{3}=-\cos \delta\right)$. Here the integrals are evaluated for each panel extending between the two vertices at $\left(y_{m}, z_{m},\right)$ and $\left(y_{m+1}, z_{m+1}\right)$. The number of panels on each 2D section is denoted by $N_{p}$. Similarly the line integral over the vessel's length $L$ is also computed using a piece-wise analytical integration procedure. In the present framework, this integration is carried out simply by assuming a linear relation between the values of two consecutive sectional integrals evaluated at $x_{j}$ and $x_{j+1}$ as:

$$
I_{3}=\sum_{j=1}^{N_{x}-1} \int_{x_{j}}^{x_{j+1}}\left(c_{0} x+c_{1}\right) e^{\mathrm{i} K x \cos \beta} d x,
$$

where $N_{x}$ is number of $2 \mathrm{D}$ sections along the vessel length.

\subsection{The Disturbance Potential Terms}

The $x$-derivatives of the disturbance velocity potentials $\psi_{B}$ and the normal vectors, which appear in the terms $Q$ and $M$ in Equation (2.18), are computed by the method proposed by [34]. This scheme applies a relation from differential geometry (see for example [35]) to express the Cartesian gradient of the potential on the body surface as a combination of the surface gradient and the derivative in the normal direction. Taking $u$ and $v$ as parametric coordinates in the longitudinal and vertical directions along the hull surface, we write

$$
\nabla \phi=\nabla_{s} \phi+\vec{n} \frac{\partial \phi}{\partial n}, \quad \nabla_{s} \equiv \frac{1}{H^{2}}\left[\vec{x}_{u}\left(G \frac{\partial}{\partial u}-F \frac{\partial}{\partial v}\right)+\vec{x}_{v}\left(E \frac{\partial}{\partial v}-F \frac{\partial}{\partial u}\right)\right] .
$$

Here the subscripts indicate partial differentiation with respect to the parametric variables, $H=$ $\sqrt{E G-F^{2}}, \vec{n}=\left(\vec{x}_{u} \times \vec{x}_{v}\right) / H$, and $E, F, G$ are the coefficients of the first fundamental form of the surface given by

$$
E=\vec{x}_{u} \cdot \vec{x}_{u}, \quad F=\vec{x}_{u} \cdot \vec{x}_{v}, \quad G=\vec{x}_{v} \cdot \vec{x}_{v} .
$$

For the velocity potentials, the normal derivatives are known from the body boundary conditions, and for the normal vector these normal derivatives are simply zero. The parametric derivatives are computed using fourth-order finite-difference schemes and the result converges quite fast with 20 to 30 two-dimensional sections along the body.

The sectional integrals over $C_{x}$ in Equation (2.18) are calculated using simple summations along the two-dimensional sections. The convergence of this integration is also quite fast and is achieved at 20 to 30 panels. In addition the line integral along the body is performed using the trapezoidal rule.

\section{Results}

According to the formulations introduced in Section 2, and for the purpose of comparison, results for added resistance are presented for the following 3 cases: 


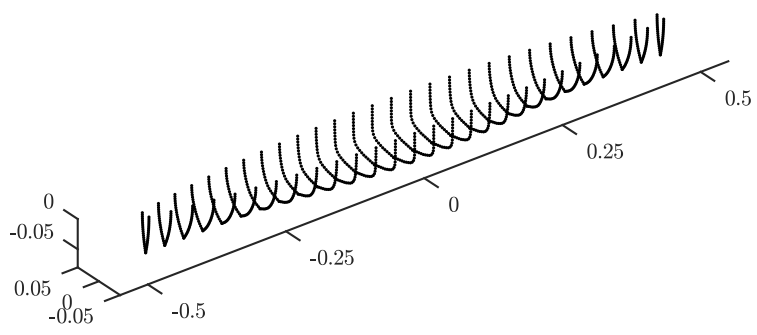

(a) Wigley Type I

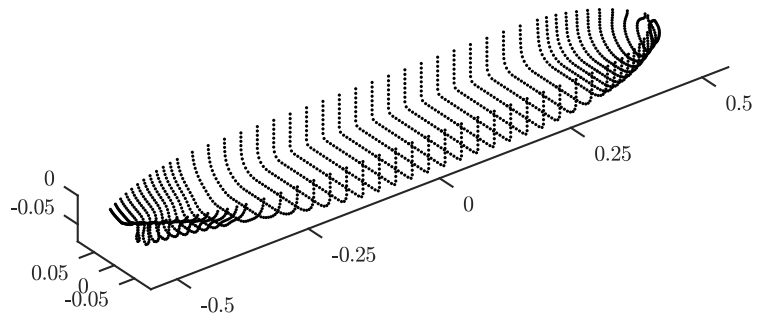

(c) KVLCC2

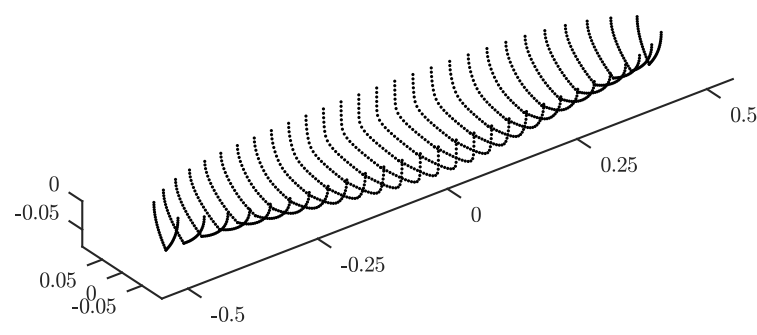

(b) Modified Wigley

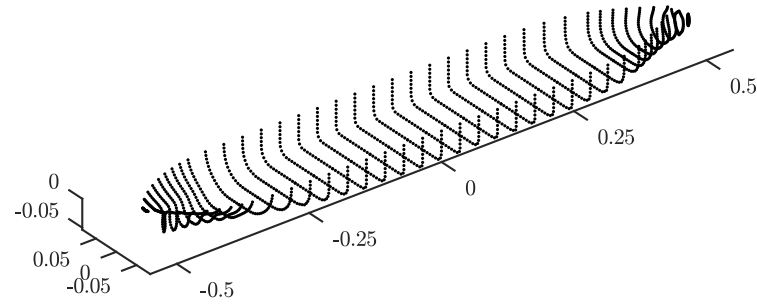

(d) RIOS Bulk Carrier

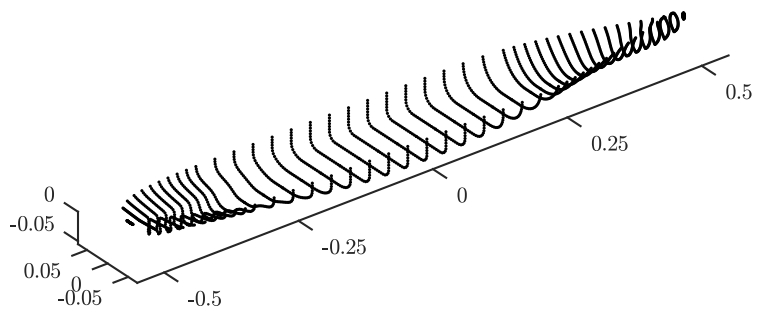

(e) DTC Container Ship

Figure 2: The geometry of the ship hulls and the strip discretisations

1. The results based on only (2.13), together with the long-wave assumptions (2.15), (2.16). Note this method is the one which has been introduced by Salvesen and is being used currently by the community. In this paper these results are denoted by Salvesen (App.). Here "App." indicates the fact that the Kochin Function in (2.13) is calculated using the long-wave approximations.

2. The results based on only (2.13), when the related Kochin Function is calculated instead by direct piece-wise analytical integration of the disturbance potentials over the sections. These results are denoted by Salvesen (Dir.).

3. The results based on both (2.13) and (2.18), where the Kochin Function is computed by direct piece-wise integration (as in case 2), and in addition the body disturbance term is taken in to account by the direct calculation of the $x$ derivative of the potentials and the normal vectors. The results using this procedure are denoted simply as Salvesen (New).

The results according to above-mentioned three methods are presented for five geometries, all shown in Figure 2. Wherever possible they are compared with experimental measurements or with solutions based on other methods. The results using Maruo's formulation inside the STF strip theory [30], are denoted as STF-Maruo. For the wave drift force $F r=0$, the solution by WAMIT [36] is also shown. Note also for the results here, only the heave and pitch modes are included. 

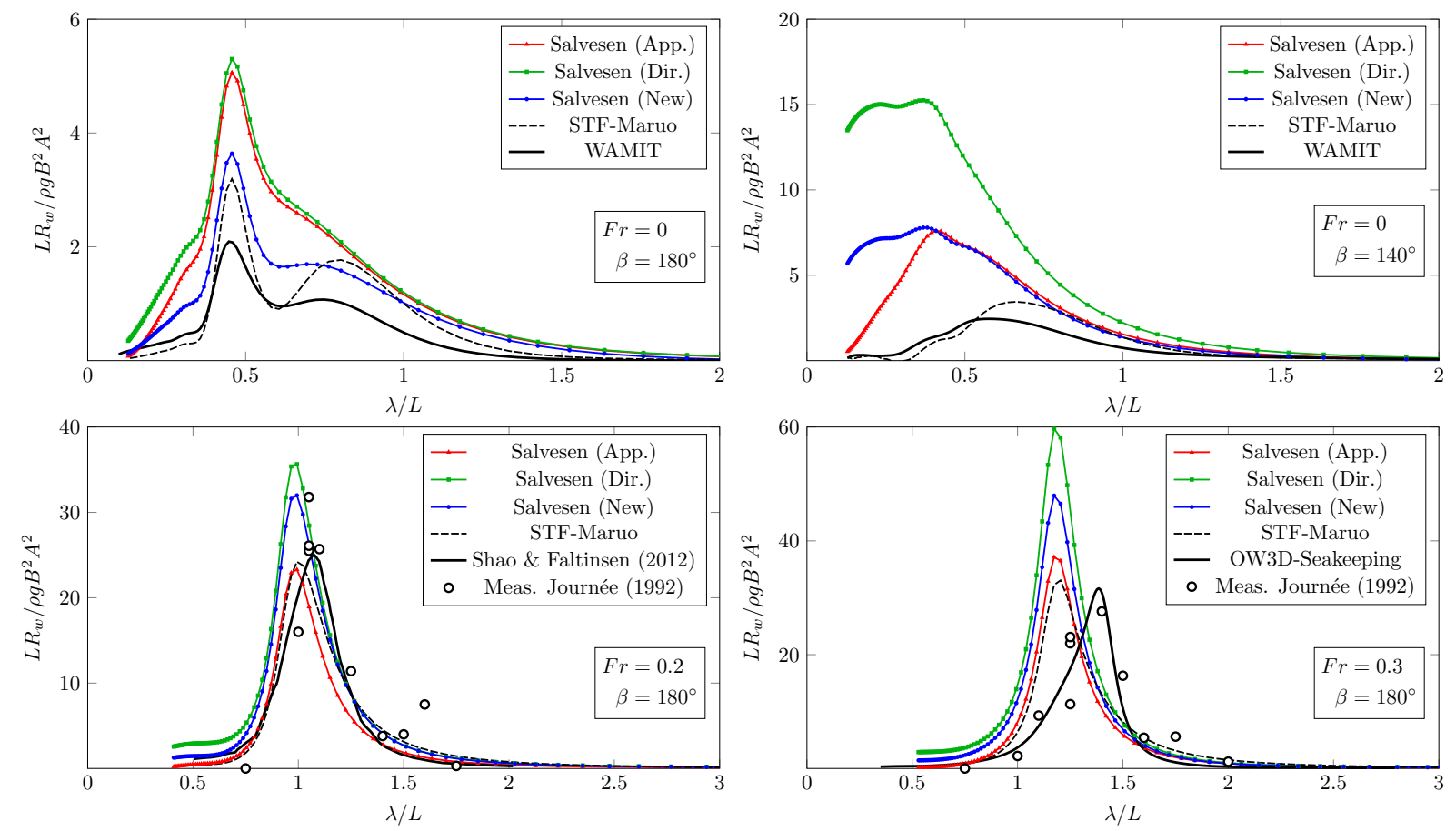

Figure 3: The wave drift force and the added resistances of the Wigley hull (I).

\subsection{Geometry 1 - Wigley Hull Type I}

Figure 3 presents the results for the wave drift force $(F r=0)$ and the added resistance $(F r=0.2,0.3)$, for the Wigley hull Type I. The results based on the 3D Boundary Element Method by by Shao \& Faltinsen in [37] is shown for $F r=0.2$. For $F r=0.3$, the added resistance calculated using the 3D finite-difference model of Amini-Afshar \& Bingham (OWD3D-Seakeeping) [38, 39, 40] is also presented for the comparison. 

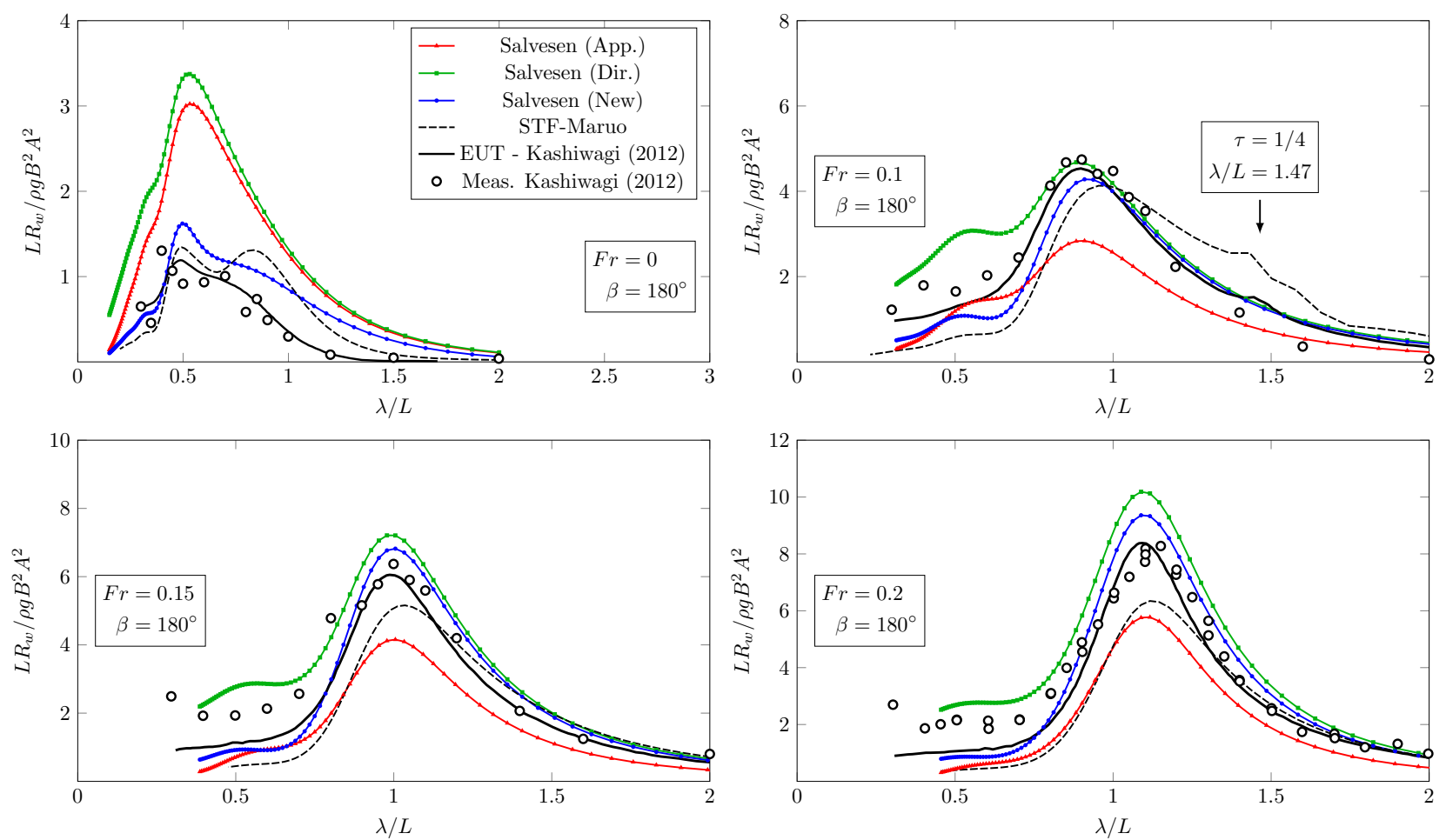

Figure 4: The wave drift force and the added resistances of the modified Wigley hull.

\subsection{Geometry 2 - Modified Wigley}

The results for the Modified Wigley hull are shown in Figure 4, for a range of Froude numbers $F r=0,0.1,0.15,0.2$. The geometry, the experimental measurements and the solutions based on Enhanced Unified Theory EUT are all from Kashiwagi et al. in [12]. 

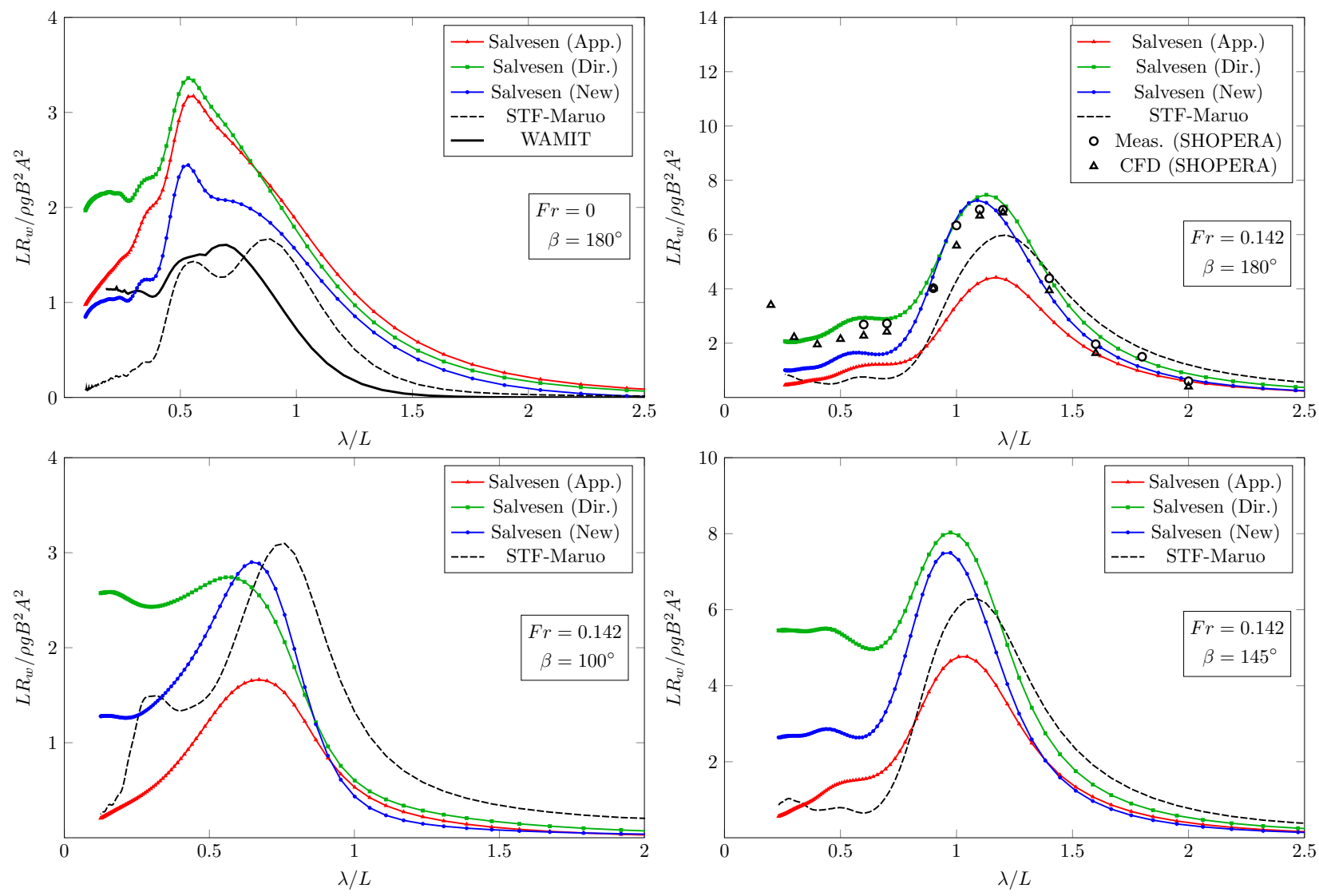

Figure 5: The wave drift force and the added resistance of the KVLCC2 hull.

\subsection{Geometry 3 - KVLCC2}

The well-known geometry of KVLCC2 is also considered. In figure 5 the wave drift force $(F r=0)$ and the added resistance at $F r=0.18$ are shown. The experimental data and the CFD calculations are from the results of the SHOPERA project in [41, 42]. 

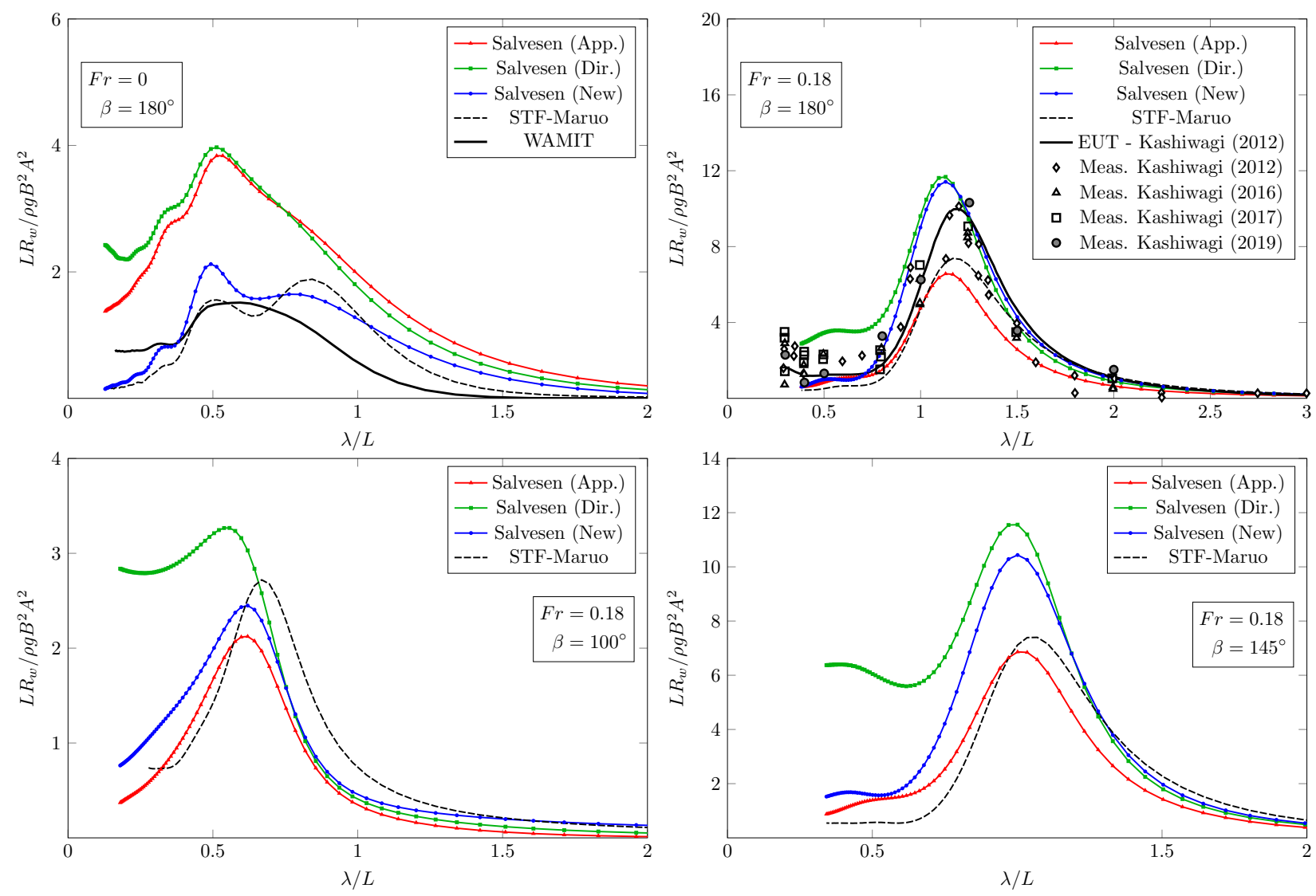

Figure 6: The wave drift force and the added resistance for the RIOS bulk carrier.

\subsection{Geometry 4 - RIOS Bulk Carrier}

The results for the RIOS bulk carrier [43] are presented in Figure 6, for the wave drift force $\mathrm{Fr}=0$ and the added resistance at $F r=0.18$. The experimental measurements in the plot are denoted as Kashiwagi (2012), Kashiwagi (2016) and Kashiwagi (2017), see [44]. The most recent experimental data which is based on a novel approach and uses the unsteady pressure measurement technique, are due to Kashiwagi in [45]. 


\subsection{Geometry 5 - DTC Container}

Next the Duisburg Test Case (DTC) container ship is considered [46]. The dimensions of the container ship in this case are $L=355 \mathrm{~m}, B=51.0 \mathrm{~m}$ and the design draft $d=14.5 \mathrm{~m}$. The results are for $F r=0.05, F r=0.11$ and $F r=0.20$. See Figure 2e for the shape and the strip discretisation of this vessel. The measurements and the CFD calculations are by Sigmund \& el Moctar from [47].
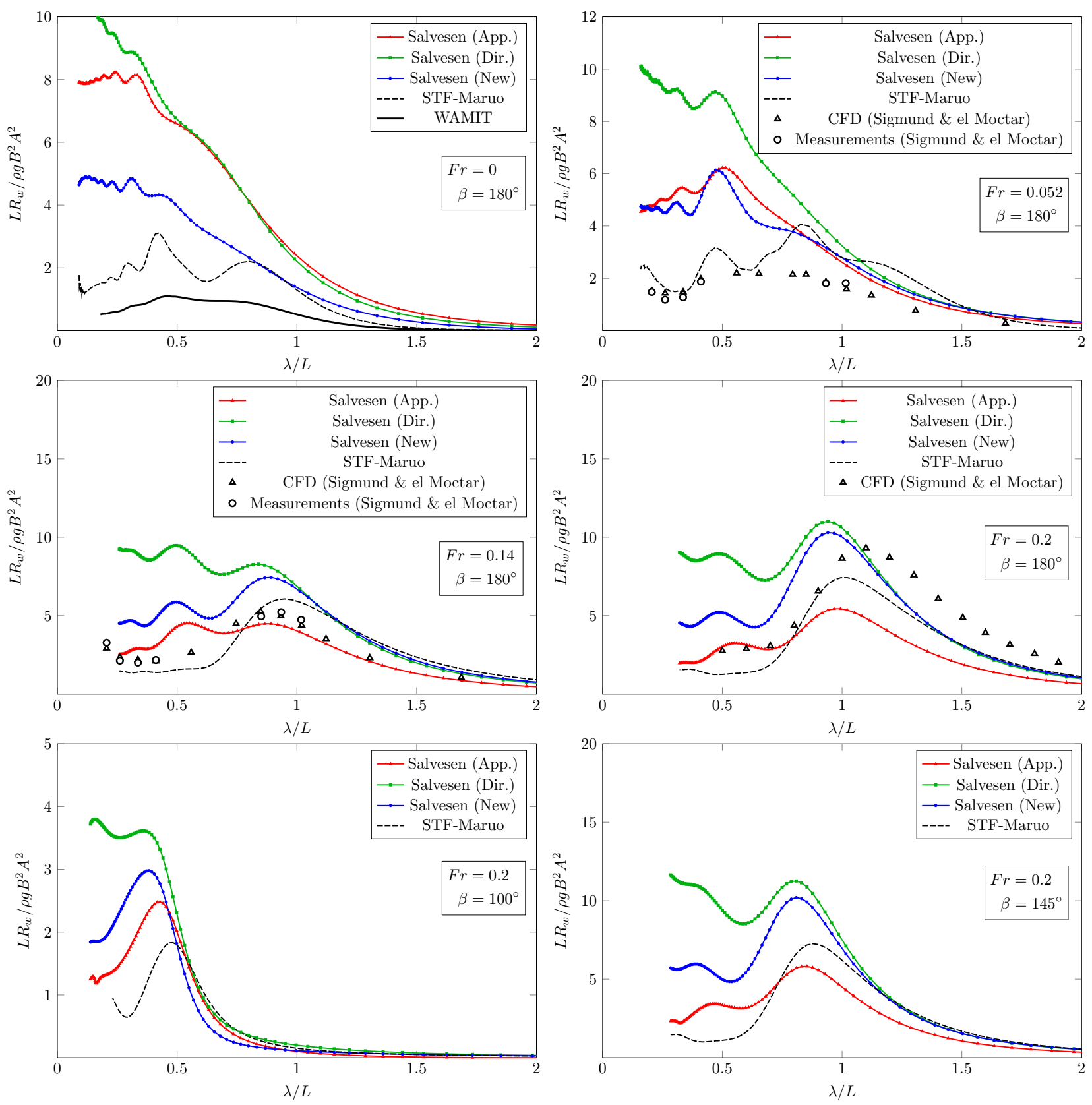

Figure 7: The wave drift force and the added resistance of the DTC container ship. 


\subsection{Discussion}

First note that in order to find the contribution of the disturbance potential term, the results denoted by Salvesen (Dir.) and Salvesen (New) should be compared. In addition, to find the validity of the long-wave assumption, the results named Salvesen (App.) should be compared with the results denoted as Salvesen (Dir.).

The results presented for these five geometries all depict the same picture about the essence of the terms in Equation (2.6). As mentioned before, and can be observed from the plots, the long-wave assumptions are generally valid only for the zero-speed case in the head-seas condition. This is why the head-seas drift forces calculated based on Salvesen (Dir.) and Salvesen (App.) are more or less the same, though both tend to dramatically over-predict the force compared with the reference solution from WAMIT. In addition, as shown in the case of the Wigley Hull for $F r=0, \beta=140^{\circ}$, the long-wave assumption also breaks down for zero-speed problems if the heading angle is other than $180^{\circ}$. For all of these zero-speed cases, the computation based on the exact implementation i.e. Salvesen (New) are remarkably closer to the WAMIT or other reference solutions. Moreover, a comparison between the results from Salvesen (Dir.) and Salvesen (New), reveals that the body disturbance term (2.17) has a significant contribution to the final zero-speed results. For the forward-speed cases (added resistance), as also recently demonstrated by [30], the long-wave assumption is no longer valid. This again can be seen by comparing the original results based on Salvesen's method (denoted as Salvesen (App.)) with the results using the direct computation of the Kochin Function in Equation (2.13), (i.e. those denoted by Salvesen (Dir.)). The role of the body disturbance term is also important here in order to be able to predict the added resistance accurately. Ignoring these body potential terms leads to over-prediction of the results especially for the high-frequency ranges. The results based on the complete form of Equation (2.6) with no long-wave or weak-scatterer assumptions (denoted as Salvesen (New) in the plots), agree considerably better with the experimental measurements. As is well known, the approximate Salvesen's method over-predicts the zero-speed drift forces, while it under-predicts the added resistance. It is shown in this paper that both of these shortcomings are remedied by using the complete implementation of Salvesen's original formulation. Moreover, as can be seen from the figures, the Salvesen (New) results compare with the experiments even better than those based on STF-Maruo from [30]. No experimental measurements were available to present here for the added resistance of these geometries in beam- or near-beam seas conditions. But from the figures it can be seen that neither the long-wave nor the weak-scatterer assumption are acceptable also in these cases.

\section{Conclusions}

Salvesen in [2], [3], mentions that the neglect of the body disturbance term related to $\nabla \Phi_{B}$ would lead to less accurate results in zero-speed and beam- or near-beam seas conditions, however no serious question was raised about the validity of the long-wave assumptions (2.15) and (2.16) in computing the wave drift forces and added resistance. In addition, to the knowledge of the author, no attempt has been made to investigate the influence of the body potentials on the wave drift force and added resistance. Therefore in this study the original equation derived by Salvesen in 1974 has been revisited. The objective was to investigate the validity of both of these assumptions, in terms of the approximate expressions for computing the second-order quantities. An implementation of STF strip theory using a low-order Boundary Element Method and the free-surface Green function has been used. Using the resultant solutions for the radiation and the scattering velocity potentials over the two-dimensional sections along the body length, Equations (2.13) and (2.17) have been calculated in their original form. The results confirm that neither the long-wave nor the weak-scatterer assumptions are generally acceptable, and good agreement with measurements is achieved when the body disturbance part is included and at the same time the Kochin Function is computed without a long-wave approximation. In fact it is proved in this paper that the role of the body disturbance term is not a matter of relative importance for some wave conditions or geometries. The body disturbance term related to $\nabla \Phi_{B}$ is in reality a fundamental part of the equation for the added resistance (2.6), and regardless of the geometry or the wave condition, the correct results are attained only after incorporating this part 
into the equation. More importantly Salvesen's approximate relations provide reasonable results, only because the long-wave assumption tends to partially correct for the neglect of the body disturbance terms. If Salvesen had computed the Kochin Function in (2.13) without the long-wave assumptions, he would have achieved the results denoted here by Salvesen (Dir.), which considerably over-predict the forces, especially in the short-wave range.

The Kochin Function related to the horizontal drift force in (2.14) can also be calculated directly and without the long-wave assumption. In addition, the related body disturbance term $\nabla \Phi_{B}$ can be included by the direct computation of the $y$ derivatives of the velocity potentials and the normal vectors. A similar picture about the accuracy of the results for the horizontal drift force can be expected. The exact equation for the mean second-order yaw moment has also been derived by Salvesen in [2]. Based on the long-wave and weak-scatterer assumptions, he presented approximate relations for computing also these second-order quantities. Therefore the same methodology shown in this paper for the wave drift force and added resistance, can be adopted for accurate computation of the mean second-order yaw moments.

Maruo's formulation, as applied to STF theory in [30], requires multiple direct calculations of the Kochin Function integral at each frequency, while the presented methodology in this paper needs only one calculation. However it requires that the body disturbance terms are taken in to account via a direct computation of the derivatives of the potentials and the normal vectors. This extra computational burden is worth considering the influence of the disturbance velocity potentials on the accuracy of the results denoted by Salvesen (New) in this paper.

\section{Acknowledgment}

I am deeply indebted to Professor Harry B. Bingham (my PhD and post-doc advisor at the Technical University of Denmark), from whom and in whose school of thought, I have learned the fascinating subject of marine hydrodynamics. In addition I am especially grateful to him for his valuable support, comments and suggestions regarding this paper. 


\section{References}

[1] N. Salvesen, E. O. Tuck, and O. Faltinsen, Ship motions and sea loads. Annual Meeting of the Society of Naval Architecture and Marine Engineers (SNAME), New York, November 12-13, 1970.

[2] N. Salvesen, "Second-order steady state forces and moments on surface ships in oblique regular waves," in International Symposium on Dynamics of Marine Vehicles and Structures in Waves, Paper, vol. 22, 1974.

[3] N. Salvesen, "Added resistance of ships in waves," Journal of Hydronautics, vol. 12, no. 1, pp. 2434, 1978.

[4] H. B. Bingham and M. Amini-Afshar, "DTU_StripTheorySolver," 2020. https://gitlab.gbar.dtu.dk/oceanwave3d/DTU_StripTheorySolver.git.

[5] N. Kochin, "On the wave resistance and lift of bodies submerged in fluid," in Transactions of Conference on the Theory of Wave Resistance, Moscow, 1936, 1936.

[6] F. Stern, J. Yang, Z. Wang, H. Sadat-Hosseini, M. Mousaviraad, S. Bhushan, and T. Xing, "Computational ship hydrodynamics: Nowadays and way forward," International Shipbuilding Progress, vol. 60, no. 1-4, pp. 3-105, 2013.

[7] F. Stern, Z. Wang, J. Yang, H. Sadat-Hosseini, M. Mousaviraad, S. Bhushan, M. Diez, Y. SungHwan, P.-C. Wu, S. M. Yeon, et al., "Recent progress in CFD for naval architecture and ocean engineering," Journal of Hydrodynamics, vol. 27, no. 1, pp. 1-23, 2015.

[8] M. Kjellberg, Fully Nonlinear Unsteady Three-Dimensional Boundary Element Method for Ship Motions in Waves. PhD thesis, Chalmers University of Technology, 2013.

[9] K.-H. Kim and Y. Kim, "Numerical study on added resistance of ships by using a time-domain Rankine panel method," Ocean Engineering, vol. 38, no. 13, pp. 1357-1367, 2011.

[10] S. A. G. Joncquez, H. B. Bingham, P. Andersen, and D. Kring, "Validation of added resistance computations by a potential-flow boundary-element method," in Symposium on Naval Hydrodynamics, Office of Naval Research, USA, 2008.

[11] M. Amini-Afshar, H. B. Bingham, W. Henshaw, and R. Read, "Convergence of near-field added resistance calculations using a high-order finite-difference method," in Proceedings of 13th PRADS, 2016.

[12] M. Kashiwagi, T. Ikeda, T. Sasakawa, et al., "Effects of forward speed of a ship on added resistance in waves," International Journal of Offshore and Polar Engineering, vol. 20, no. 3, pp. 196-203, 2010 .

[13] M. Kashiwagi, "Numerical seakeeping calculations based on the slender ship theory," Ship Technology Research (Schiffstechnik), vol. 4, no. 4, pp. 167-192, 1997.

[14] M. Kashiwagi, "Added resistance, wave-induced steady sway force and yaw moment on an advancing ship," Ship Technology Research (Schiffstechnik), vol. 39, no. 1, pp. 3-16, 1992.

[15] M. Kashiwagi, "Prediction of surge and its effect on added resistance by means of the enhanced unified theory," in Transactions of the West-Japan Society of Naval Architects 89, pp. 77-89, The Japan Society of Naval Architects And Ocean Engineers, 1995.

[16] J. N. Newman, "The theory of ship motions," Advances in Applied Mechanics, vol. 18, pp. 221$283,1979$.

[17] J. Newman and P. Sclavounos, "The unified theory of ship motions," in 13th Symposium on Naval Hydrodynamics, (Tokyo), pp. 373-394, 1980. 
[18] T. F. Ogilvie and E. O. Tuck, "A rational strip theory of ship motions: Part I," tech. rep., University of Michigan, 1969.

[19] O. Faltinsen, "A rational strip theory of ship motions: Part II," tech. rep., University of Michigan, 1971.

[20] P. Sclavounos, "The diffraction of free-surface waves by a slender ship," Journal of Ship Research, vol. 28, no. 01, pp. 29-47, 1984.

[21] J. Newman, "A slender-body theory for ship oscillations in waves," Journal of Fluid Mechanics, vol. 18 , no. 4, pp. 602-618, 1964.

[22] J. N. Newman and E. O. Tuck, "Current progress in the slender body theory for ship motions," in Proceedings of the 5th Symposium on Naval Hydrodynamics, ONR, Bergen, Norway, pp. 129-166, Paper: 1964-2 Proceedings., 1964.

[23] P. D. Sclavounos, "The unifed slender-body theory: Ship motions in waves," in Proc. 15th Symp. on Naval Hydrodyn. Hamburg, pp. 177-192, 1985.

[24] H. Maruo, "Wave resistance of a ship in regular head seas," Bulletin of the Faculty of Engineering, Yokohama National University, vol. vol. 9, 1960.

[25] H. Maruo, "Resistance in waves," Research on Seakeeping Qualities of Ships in Japan, The Society of Naval Architects of Japan, vol. 8, pp. 67-102, 1963.

[26] J. Gerritsma and W. Beukelman, "Analysis of the resistance increase in waves of a fast cargo ship," International Shipbuilding Progress, vol. 19, no. 217, 1972.

[27] J. Gerritsma and W. Beukelman, "Analysis of the modified strip theory for the calculation of ship motions and wave bending moments," International Shipbuilding Progress, vol. 14, no. 156, pp. 319-337, 1967.

[28] T. Loukakis and P. Sclavounos, "Some extensions of the classical approach to strip theory of ship motions, including the calculation of mean added forces and moments," Journal of Ship Research, vol. 22 , no. $1,1978$.

[29] W.-C. Lin and A. Reed, "The second order steady force and moment on a ship moving in an oblique seaway," in Proceedings, 11th Symposium on Naval Hydrodynamics, London, Great Britain, pp. 333-351, 1976.

[30] M. Amini-Afshar and H. B. Bingham, "Added resistance using Salvesen-Tuck-Faltinsen strip theory and the kochin function," Applied Ocean Research, vol. 106, p. 102481, 2021.

[31] M.-C. Fang, "Second-order steady forces on a ship advancing in waves," International shipbuilding progress, vol. 38, no. 413, pp. 73-93, 1991.

[32] M.-C. Fang and G.-R. Chen, "On the nonlinear hydrodynamic forces for a ship advancing in waves," Ocean Engineering, vol. 33, no. 16, pp. 2119-2134, 2006.

[33] J. Newman, "The interaction of stationary vessels with regular waves," in Proceedings of the 11th Symposium on Naval Hydrodynamics, London, 1976, 1976.

[34] H. B. Bingham and H. Maniar, "Computing the double-body m-terms using a B-spline based panel method," in Proceedings of the 11th international workshop on water waves and floating bodies, 1996, 1996.

[35] L. Brand, Vector and tensor analysis. Courier Dover Publications, 2020.

[36] C.-H. Lee, WAMIT theory manual. Massachusetts Institute of Technology, Department of Ocean Engineering, 1995. 
[37] Y.-L. Shao and O. M. Faltinsen, "Linear seakeeping and added resistance analysis by means of body-fixed coordinate system," Journal of Marine Science and Technology, vol. 17, no. 4, pp. $493-510,2012$.

[38] M. Amini-Afshar, H. B. Bingham, and W. D. Henshaw, "Stability analysis of high-order finitedifference discretizations of the linearized forward-speed seakeeping problem," Applied Ocean Research, vol. 92, p. 101913, 2019.

[39] M. Amini-Afshar and H. B. Bingham, "Pseudo-impulsive solutions of the forward-speed diffraction problem using a high-order finite-difference method," Applied Ocean Research, vol. 80, pp. 197$219,2018$.

[40] M. Amini-Afshar and H. B. Bingham, "Solving the linearized forward-speed radiation problem using a high-order finite difference method on overlapping grids," Applied Ocean Research, vol. 69, pp. 220-244, 2017.

[41] A. Papanikolaou, G. Zaraphonitis, E. Bitner-Gregersen, V. Shigunov, O. El Moctar, C. G. Soares, D. Reddy, and F. Sprenger, "Energy efficient safe ship operation (SHOPERA)," Transportation Research Procedia, vol. 14, pp. 820-829, 2016.

[42] V. Shigunov, O. el Moctar, A. Papanikolaou, R. Potthoff, and S. Liu, "International benchmark study on numerical simulation methods for prediction of manoeuvrability of ships in waves," Ocean Engineering, vol. 165, pp. 365 - 385, 2018.

[43] H. Iwashita, M. Kashiwagi, Y. Ito, and Y. Seki, "Calculations of ship seakeeping in low-speed/lowfrequency range by frequency-domain Rankine panel methods," Journal of the Japan Society of Naval Architects and Ocean Engineers, vol. 24, pp. 129-146, 2016, (In Japanese).

[44] H. Iwashita and M. Kashiwagi, "An innovative EFD for studying ship seakeeping," Proceedings of 33rd IWWWFB (Guidel-Plages, France), pp. 85-88, 2018.

[45] M. Kashiwagi, H. Iwashita, S. Miura, and M. Hinatsu, "Study on added resistance with measured unsteady pressure distribution on ship-hull surface," Proceedings of 34th IWWWFB (Noah's on the Beach, Newcastle, NSW, Australia), pp. 81-84, 2019.

[46] B. O. el Moctar, V. Shigunov, and T. Zorn, "Duisburg test case: Post-panamax container ship for benchmarking," Ship Technology Research, vol. 59, no. 3, pp. 50-64, 2012.

[47] S. Sigmund and B. O. el Moctar, "Numerical and experimental investigation of added resistance of different ship types in short and long waves," Ocean Engineering, vol. 147, pp. 51-67, 2018. 